\title{
Examining Energy Information Literacy with an Adaptation of the Everyday Health Information Literacy Screening Tool
}

\author{
Teija Keränen ${ }^{1}$, Noora Hirvonen ${ }^{1,2}$, and Maija-Leena Huotari, \\ Information Studies, University of Oulu \& Medical Research Center, Oulu, \\ Finland \\ Information Studies, Åbo Akademi University, Turku, Finland \\ \{teija.keranen, noora.hirvonen, maija- \\ leena.huotari\}@oulu.fi
}

\begin{abstract}
This study explores energy information literacy by applying an adapted version of an everyday health information literacy (EHIL) screening tool. For this study, the original EHIL tool was modified by adjusting its ten statements to an energy context. Data were collected with an online survey from students present for the academic year in 2016-2017 $(\mathrm{n}=11,381)$ at the University of Oulu. Statistical analyses include an exploratory factor analysis and comparison of mean factor scores. Survey items on respondents' background were also included in the analyses. The response rate was 12.2 percent $(n=1,390)$. The screening tool's factorial structure was found to be multifaceted and to resemble that of the original EHIL tool with three factors: motivation ('motivation'), confidence in finding energy information ('confidence'), and perceived ability to evaluate it ('evaluation'). Mean factor scores varied by gender and field of study. The study is among the first to examine energy information literacy.
\end{abstract}

Keywords: Energy information literacy, screening tool, everyday life, health information literacy, online survey.

\section{Introduction and Objectives}

\subsection{The Concept of Energy Information Literacy}

Transitions in energy production and consumption are currently taking place on many levels of society. At the same time, global energy demand is increasing and there is a need to mitigate climate change by reducing greenhouse gas emissions. Cleaner, more energy efficient technologies have been developed in recent years and governments have made efforts towards more sustainable paths in energy production [1]. Energy related decisions are made not only on a energy market or policy levels but also on an individual level through choices people make in their everyday lives when acting as energy consumers and, increasingly, producers. The understanding that people develop on energy issues guides these decisions [2]. In this study, we focus on people's abilities 
to seek, evaluate, and use energy related information in everyday situations and introduce the concept of energy information literacy (EIL). Furthermore, we explore the applicability of an everyday health information literacy (EHIL) screening tool adapted to an energy context.

The concept of EIL is connected to energy literacy (EL) which refers to people's skills to make informed energy-related choices. A broadly used definition of EL by DeWaters and Powers [3] consists of cognitive (knowledge), affective (attitudes and values), and behavioural aspects. DeWaters and Powers state that an energy literate individual "has a sound conceptual knowledge base as well as a thorough understanding of how energy is used in everyday life, understands the impact that energy production and consumption have on all spheres of our environment and society, is sympathetic to the need for energy conservation and the need to develop alternatives to fossil fuelbased energy resources, is cognizant of the impact that personal energy-related decisions and actions have on the global community, and - most importantly - strives to make choices and exhibit behaviors that reflect these attitudes with respect to energy resource development and energy consumption" [3, p. 45]. Another related concept is green information literacy which refers to conventional information literacy skills expanded to include sustainable thinking, that is, considering how information behavior, information choices, and information actions influence the environment [4].

Our examination of the concept of energy information literacy is based on an application of the concept of health information literacy that was introduced in the early the 2000 's. It was defined as "the set of abilities to recognize a health information need, identify likely information sources and use them to retrieve relevant information, assess the quality of the information and its applicability to a specific situation, and analyze, understand, and use the information to make good health decisions" [5, p. 294]. The definition was framed within two related concepts: health literacy and information literacy. In a similar manner, we propose that the concept of energy information literacy may be used to refer to information literacy in an energy context: to abilities needed to recognize an energy-related information need; identify likely information sources and use them to retrieve relevant information; assess the quality of the information and its applicability to a specific situation; and analyze, understand, and use the information to make good decisions in terms of energy.

While energy literacy refers to energy-raleted knowledge, attitudes, and intentions or behavior [3], energy information literacy emphasizes the meta-cognitive abilities that form the basis for lifelong learning such as being able to locate relevant information and to evaluate and use it effectively (see [6]). These abilities can be considered especially important in the contemporary, constantly changing information environment. For a long time, the concept of information literacy was mainly applied in educational settings. More recently, the focus of information literacy research has expanded to everyday life context as well. Yet, there is still a significant gap in this area of research [7].

\subsection{Assessment of Energy Information Literacy}

Energy literacy has been assessed based on self-reported behaviour and energy literacy 
tests, namely, what people say, do, and know [8]. Mainly it has been evaluated quantitatively and from the perspective of rational decision-making in energy related issues [9] or as the ability to evaluate energy use of household appliances [10]. Target groups have been, for example, middle school and high school students [3] or households [9].

In the context of health, Niemelä, Ek, Eriksson-Backa and Huotari [11] designed an everyday health information literacy (EHIL) screening tool based on the Medical Library Association's definition of health information literacy. The screening tool was meant to detect individuals or groups with problems related to their interest and motivation, finding, understanding, evaluating, or using health information, but being literate at the average level (being able to read). The tool consist of ten statements (EHIL1-EHIL10) to which participants are asked to respond in a scale from 1 (strongly disagree) to 5 (strongly agree). The tool was found to have a multifaceted structure with three factors: motivation to seek information ('motivation', EHIL1, EHIL3, EHIL2, EHIL9), confidence in finding information ('confidence', EHIL10, EHIL8, EHIL5, EHIL4), and perceived abilities to evaluate information ('evaluation', EHIL7, EHIL6) $[11,12]$.

\subsection{Objectives}

To our knowledge, this study is among the first to examine energy information literacy. It aims at exploring energy information literacy by applying an adapted version of the EHIL screening tool. The research questions are set as follows:

Q1: What is the factorial structure of the everyday energy information literacy (EEIL) screening tool?

Q2: Do mean factor scores vary across respondents' background variables?

\section{Method}

\subsection{Data Collection}

The data were collected with an online survey sent to all students present for the academic year 2016-2017 at the University of Oulu, Finland, in January 2017 $(n=11,381)$. The response rate was 12.2 percent $(n=1,390)$. The original EHIL screening tool was modified to an everyday energy information literacy (EEIL) screening tool by adjusting its ten statements to an energy context:

EEIL1. It is important to be informed about energy issues.

EEIL2. I know where to seek energy information.

EEIL3. I like to get energy information from a variety of sources.

EEIL4. It is difficult to find energy information from printed sources (magazines and books).

EEIL5. It is difficult to find energy information from the Internet.

EEIL6. It is easy to assess the reliability of energy information in printed sources (magazines and books). 
EEIL7. It is easy to assess the reliability of energy information on the Internet.

EEIL8. Energy related terminology and statements are often difficult to understand. EEIL9. I apply energy related information to my own life and/or that of people close to me.

EEIL10. It is difficult to know who to believe in energy issues.

The respondents were provided with a short definition of energy by explaining that, in this context, energy refers to energy used by households mainly for lighting and warming as well as energy used by traffic and industry.

Also, questions gathered demographic items on the respondents' backgrounds. The respondents were asked about their year of birth, gender, highest education, work situation, income, major field of study, and whether their studies relate to the energy industry or electricity market. Further, they were asked about their place of residence, the type of house or appartment they lived in, home ownership, and living arrangements.

\subsection{Data Analysis}

The internal consistency of the 10-item everyday energy information literacy screening tool was analysed using unstandardised Cronbach's alpha. The principal component analysis was chosen as the extraction method in the exploratory factor analyses because the aim was to describe the factorial structure of the screening tool. According to the Kaiser-Guttman-criterion, all factors with an eigenvalue of $>1$ were extracted. To improve the interpretability of the extracted factors, an orthogonal rotation (Varimax criterion) technique was applied, yielding statistically independent factors.

Mean factor scores were calculated with the regression method, and one-way analyses of variance (ANOVA), and independent samples t-tests were performed to examine whether the mean factor scores varied across demographic variables and respondents' field of study. The relationship between factor scores and respondents' background variables were analysed with analysis of variance (ANOVA) using standardized factor scores where the mean is set to 0 and standard deviation to 1 . Thus, a negative value refers to below the mean and a positive value to above the mean factor score. Standardized mean factor scores are presented in homogenous subsets: each subset comprises a set of means that do not differ from each other significantly (Tables 3 and 4 in the Results section). Statistical analyses were performed using the IBM Statistical Package for the Social Sciences (SPSS) version 22.0.

\section{$3 \quad$ Results}

\subsection{Respondents' Characteristics}

The respondents' mean age was 28.1 years and 57.0 percent $(n=785)$ were female. The majority of the repondents were full-time students ( $n=923,66.5$ percent), lived in a city with a population over $50,000(n=1212,87.3$ percent $)$, lived in a rental apartment 
$(\mathrm{n}=1078,77.6$ percentand had less than a 2,000 euro monthly household income $(\mathrm{n}=866$, 62.6). Nearly half $(n=594,42.8$ percent) lived alone and 39.6 percent $(n=550)$ in a household of two people. The respondents had majors in the Faculty of Natural Sciences $(n=249,17.9$ percent), Humanities $(n=229,16.5$ percent), Educational Sciences ( $n=214,15.4$ percent), Medicine ( $n=206,14.8$ percent), Engineering $(n=179$, 12.9 percent), Information Technology and Electrical Engineering $(n=122,8.8$ percent), Business ( $n=98,7.0$ percent) and other faculties (Architecture $n=36,2.6$ percent; Biochemistry and Molecular Medicine $n=20,1.4$ pecent; Mining School $n=27$, and 1.9 percent, other $n=8,0.6$ percent). Of the respondents 13.2 percent $(n=183)$ reported to have studies related to energy industry or electricity market, and 44.7 percent $(n=621)$ already had an academic degree.

\subsection{The Factorial Strucure of the EEIL Screening Tool}

The internal consistency of the 10-item everyday energy information literacy (EEIL) screening tool was evaluated with Cronbach's alpha and it was .701. The Kaiser-MeyerOlkin measure of sampling adequacy value was .735 , indicating that the samples were adequate to consider the data as normally distributed. Barlett's test of sphericity was significant $(\mathrm{p}<.001)$. The screening tool's factorial structure was found to be multifaceted (see Table 1). Three factors were indentified and labeled as follows (labels in parenthesis): confidence in finding and understanding information ('confidence'), motivation to do so ('motivation'), and perceived ability to evaluate information ('evaluation').

Table 1. Results of exploratory factor analyses (rotated component matrix) of data collected from Finnish university school students $(n=1,390)$. For the purpose of clarity, only factor loadings $>.50$ are printed in the table.

\begin{tabular}{|c|c|c|c|c|}
\hline Component & & 1 & 2 & 3 \\
\hline \multirow{4}{*}{ 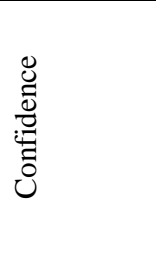 } & EEIL2 I know where to seek energy information. & -.656 & & \\
\hline & $\begin{array}{l}\text { EEIL4. It is difficult to find energy information from } \\
\text { printed sources (magazines and books). }\end{array}$ & .633 & & \\
\hline & $\begin{array}{l}\text { EEIL5. It is difficult to find energy information from } \\
\text { the Internet. }\end{array}$ & .730 & & \\
\hline & $\begin{array}{l}\text { EEIL8. Energy related terminology and statements } \\
\text { are often difficult to understand. }\end{array}$ & .637 & & \\
\hline \multirow{4}{*}{ 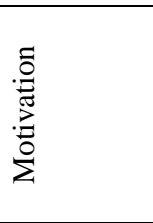 } & EEIL1. It is important to be informed about energy & & .774 & \\
\hline & issue. & & & \\
\hline & $\begin{array}{l}\text { EEIL3. I like to get energy information from a } \\
\text { variety of sources. }\end{array}$ & & .761 & \\
\hline & $\begin{array}{l}\text { EEIL9. I apply energy related information to my } \\
\text { own life and/or that of people close to me. }\end{array}$ & & .687 & \\
\hline \multirow{4}{*}{ 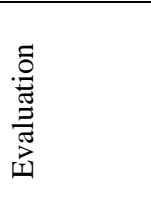 } & EEIL6. It is easy to assess the reliability of energy & & & .797 \\
\hline & $\begin{array}{l}\text { information in printed sources (magazines and } \\
\text { books). }\end{array}$ & & & \\
\hline & $\begin{array}{l}\text { EEIL7. It is easy to assess the reliability of energy } \\
\text { information on the Internet. }\end{array}$ & & & .785 \\
\hline & EEIL10. It is difficult to know who to believe in & & & -.597 \\
\hline
\end{tabular}


As can be seen in Table 2, mean factor scores were the lowest for 'evaluation' and the highest for 'motivation' in the whole sample. In further analysis, standardized mean factor scores are used (regression method).

Table 2. Descriptive statistics for the three everyday energy information literacy factors.

\begin{tabular}{llllll}
\hline & No. of items & M (SD) & Skewness & Kurtosis & Alpha \\
\hline Confidence & 4 & $3.47(.53)$ & -.124 & -.233 & .641 \\
Motivation & 3 & $3.73(.71)$ & -.408 & -.050 & .599 \\
Evaluation & 3 & $2.92(.70)$ & -.024 & -.3027 & .618 \\
\hline
\end{tabular}

\subsection{Differences by Respondents' Background}

Significant age differences were found in mean factor scores for 'motivation' and 'evaluation'. Older students (>25 years) had higher scores in 'motivation' but lower scores in 'evaluation' when compared to younger students ( $<25$ years) (see Table 3 ).

Table 3. Standardized mean factor scores (regression method) by age in homogenous subsets.

\begin{tabular}{lllll}
\hline Age & $\mathrm{N}$ & \multicolumn{5}{c}{ Subset for alpha $=0.05$} & $\mathrm{~F}, \mathrm{p}^{*}$ \\
\hline $25-30$ & 324 & .092 & Factor 1: Confidence & $1.68, .169$ \\
$23-25$ & 279 & .015 & & \\
$>30$ & 333 & -.008 & & \\
$<23$ & 433 & -.072 & & \\
\hline & & & & \\
$23-25$ & 279 & -.162 & Factor 2: Motivation & \\
$<23$ & 433 & -.093 & -.093 & \\
$25-30$ & 324 & & .049 & .049 \\
$>30$ & 333 & & & .001 \\
\hline & & & & \\
$>30$ & 333 & -.160 & Factor 3: Evaluation & \\
$25-30$ & 324 & -.004 & -.004 & .003 \\
$23-25$ & 279 & .025 & .025 & .110 \\
$<23$ & 433 & & &
\end{tabular}

*ANOVA; Tukey HSD. Uses Harmonic Mean Sample Size = 333,803; the group sizes are unequal; the harmonic mean of the group size is used; type I error levels are not guaranteed.

Male students' mean factor scores were higher in 'confidence' $(M=0.413$ vs. $M=-0.300$, $\mathrm{t}(1290)=13.653, \mathrm{p}<0.001)$ and lower in 'motivation' $(\mathrm{M}=-0.060$ vs. $\mathrm{M}=0.060$, $\mathrm{t}(1290)=2.193, \mathrm{p}=0.028)$ when compared to female students. The difference was not significant in 'evaluation' $(\mathrm{M}=0.055$ vs. $\mathrm{M}=-0.049, \mathrm{t}(1290)=-1.906, \mathrm{p}=0.066)$. 
The humanities' and educational sciences' students had the lowest and engineering students the highest mean factor scores for 'confidence' (Table 4). Natural sciences' and engineering students, and students in other fields (architecture, biochemistry and molecular medicine, mining school, and non-specified) had the highest mean factor scores both in 'evaluation' and 'motivation'. Business school and information technology and electrical engineering students, in turn, received the lowest scores in 'motivation'. Students in business school and medicine received the lowest scores in 'evaluation'.

Table 4. Mean factor scores by students' $(n=1,390)$ field of study.

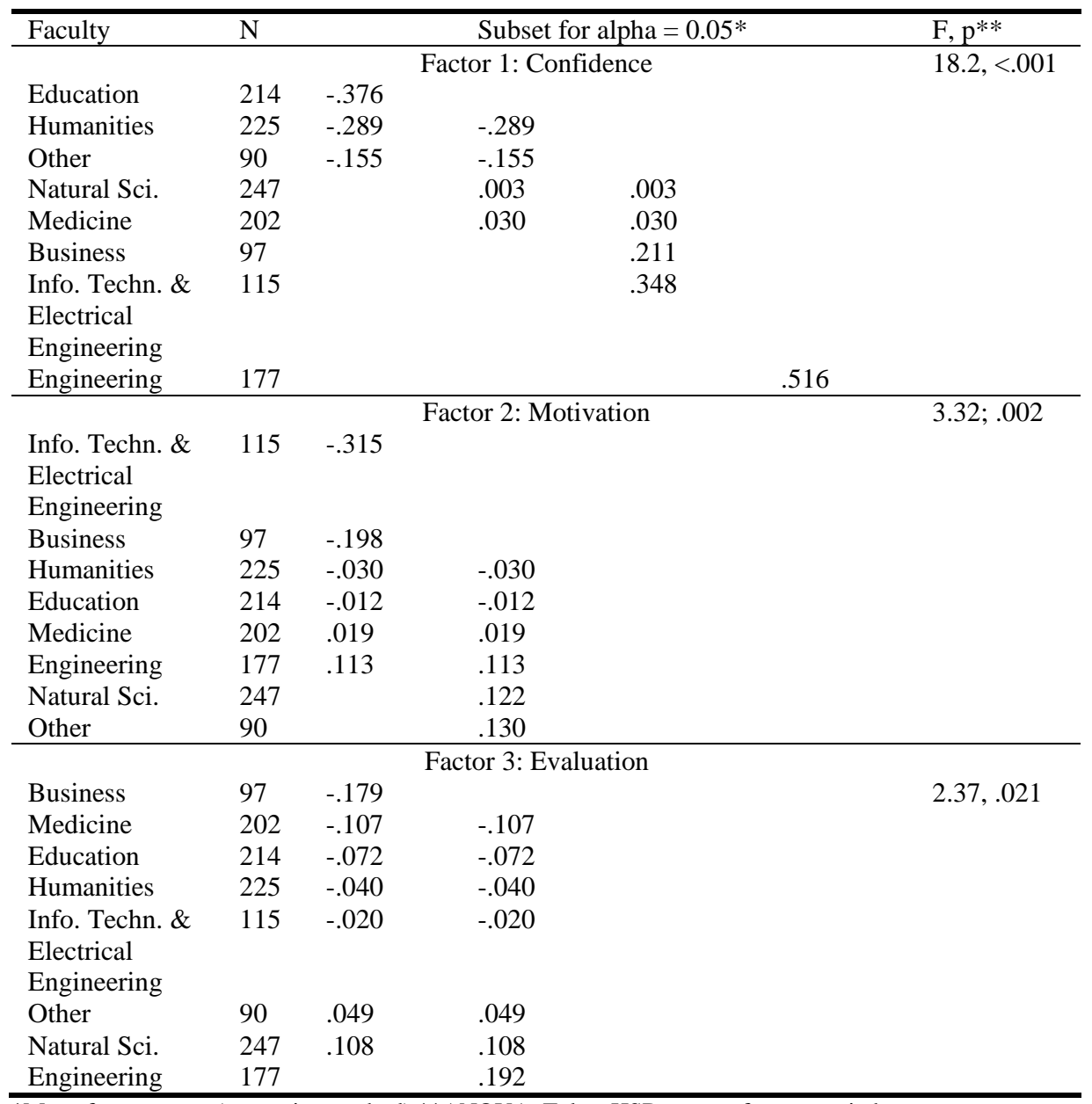

*Mean factor scores (regression method) **ANOVA; Tukey HSD, means for groups in homogenous subsets are displayed. Uses Harmonic Mean Sample Size $=333,803$; the group sizes are unequal; the harmonic mean of the group size is used; type I error levels are not guaranteed. 
Further, there were significant differences in mean scores for each factor based on whether a respondent's studies related to energy industry or electricity market or not. Those whose studies were related to these issues $(n=181)$ received higher mean factor scores in 'motivation' $(\mathrm{M}=0.245$ vs. $\mathrm{M}=-0.053, \mathrm{t}(1290)=3.728, \mathrm{p}<0.001)$, 'evaluation' $(\mathrm{M}=0.198$ vs. $\mathrm{M}=-0.023, \mathrm{t}(1290)=2.767, \mathrm{p}=0.007)$, as well as 'confidence' $(\mathrm{M}=0.312$ vs. $\mathrm{M}=-0.055, \mathrm{t}(1290)=4.630, \mathrm{p}<0.001)$.

\section{Discussion}

The everyday energy information literacy (EEIL) screening tool's factorial structure was found to be multifaceted. It resembled that of the original everyday health information literacy (EHIL) screening tool and its three factors: motivation and confidence in finding information ('motivation', 'confidence'), and perceived ability to evaluate it ('evaluation') [12]. Also some differences were found. In the original EHIL tool, EHIL2, 'I know where to seek health information', was included in 'motivation'. In the present study, in turn, a similar statement in an energy context, EEIL2, was included in the factor labeled 'confidence'. EHIL10, 'It is difficult to know who to believe in health issues', was included in 'confidence' in earlier studies in health context, whereas in the context of energy EEIL10, 'It is difficult to know who to believe in energy issues', was part of 'evaluation'.

The factor scores were found to vary by gender, age, and field of study. Older students had higher mean scores in 'motivation' and lower mean scores in 'evaluation' when compared to younger students. Similarly, in a study on everyday health information literacy, older adults were found to be more motivated to seek health information but less confident in their abilities to determine who to believe in health issues and understanding terminology when compared to younger people [13]. In a study on energy literacy by DeWaters and Powers [3], high-school students had more positive attitudes and values toward energy issues than middle-school students. However, in behavioral items the result was the opposite: middle-school students were more likely to attend to energy saving [3].

Educational level has been found to be connected to energy literacy [3] as well as to health information literacy [15] in earlier studies. In the present study, the study population was homogeneous in terms of its educational level, namely, they were all university students. However, their EEIL scores were examined based on their field of study and some differences were found. The engineering students had high mean factor scores for all three factors. Also natural sciences' students had high mean factor scores for 'evaluation' and 'motivation' as well as students categorized in the 'other' category. The humanities' and educational sciences' students, in turn, had the lowest scores for 'confidence'. The business school students had relatively low scores in both 'motivation' and 'evaluation'. Also students in information technology and electrical engineering received low scores in 'motivation', and medical students, in turn, in 'evaluation'. 
Further, significant differences were found in mean scores for each factor based on whether a respondent's studies related to energy industry or electricity market or not. These findings indicate that students whose studies involve energy related issues are more likely to be motivated to seek for energy information, and confident in their abilities to find and evaluate the information. In a study by Cotton and colleagues [14], a large proportion of students in Architecture, Design, and Environment, Geography, Earth, and Environmental Science as well as Marine Science and Engineering considered themselves as experts or quite knowledgeable in energy issues. In turn, students in Nursing and Midwifery, Social Science and Social Work, and Psychology were less confident in their energy knowledge [14].

In this study, male students' scores were higher in 'confidence' and lower in 'motivation' when compared to female students. In a similar vein, men scored higher in energy literacy tests based on knowledge [14] and women, in turn, had better scores in energy related values, attitudes, and self-efficacy [3]. Interestingly, in a study by Cotton and colleagues [14], male respondents were more likely to cite the Internet as important in contributing their understanding of energy issues, while female respondents found TV, friends, and family as important energy information sources. Also in studies on everyday health information literacy, men, and especially young men, have been found to have lower motivation than women to seek information [13]. Gender differences could be expected to explain some of the educational differences since in some fields the gender distribution tends to be rather uneven. In this study, the Educational Sciences' and Humanities' students were predominantly women and the students in Engineering and Information Technology and Electrical Engineering predominantly men. This reflects the student body at the University of Oulu. However, although male students in general were less motivated to seek for energy information than female students, engineering students received high mean factor scores in 'motivation'.

The results are indicative and should be replicated in other populations. Further, everyday energy information literacy should be studied in connection to energy consumption and production actions as well as attitudes. Information literacy is not important only in education but these competencies also guide everyday decisions. Therefore, everyday information literacy in diffent settings deserves to be examined further.

\section{Conclusions}

To our knowledge, this study is among the first to propose and examine the concept of energy information literacy (EIL). Moreover, the study contributes to further modification of the everyday health information literacy (EHIL) screening tool to other contexts, in this case, the context of energy. The factorial structure of the everyday energy information litracy screening tool is similar to that of the everyday health information literacy screening tool with three factors: motivation and confidence in finding information ('motivation', 'confidence'), and perceived ability to evaluate it ('evaluation'). Moreover, significant differences in factor scores were found based on 
respondents' background, namely, gender and field of study. These findings indicate that this screening tool has potential to be applied in the context of energy and to be further transfered to other contexts as well. The tool can be used to detect individuals or groups who lack motivation or have difficulties in finding or evaluating information on a topic. Information and guidance may be tailored to better match people's needs based on the responses to the tool's statements.

\section{Acknowledgements}

The study is funded by the Strategic Research Council, Finland, Project n:o 292854.

\section{References}

1. International Energy Agency: Energy and Climate Change. World Energy Outlook Special Report 1. (2015)

https://www.iea.org/publications/freepublications/publication /WEO2015SpecialReportonEnergyandClimateChange.pdf

2. Stern, P.C.: Individual and Household Interactions with Energy Systems: Toward Integrated Understanding. Energy Research \& Social Science 1, 41-48 (2014)

3. DeWaters, J.E.; Powers, S.E.: Energy Literacy of Secondary Students in New York State (USA): A Measure of Knowledge, Affect, and Behavior. Energy Policy 39(3), 1699-1710 (2011)

4. Kurbanoglu, S.; Boustany, J.: From Green Libraries to Green Information Literacy. Communications in Computer and Information Science 492, 47-58.

5. Shipman, J.P.; Kurtz-Rossi, S.; Funk, C.J.: The Health Information Literacy Research Project. Journal of Medical Library Association 97(4), 293-301 (2009)

6. American Library Association: Presidential Committee on Information Literacy: final report (1989) http://www.ala.org/acrl/publications/whitepapers/presidential

7. Martzoukou, K.; Sayyad, A.E.: Towards an Everyday Life Information Literacy Mind-Set: a Review of Literature. Journal of Documentation (in press)

8. Sovacool, B.K.; Blyth, P.L: Energy and Environmental Attitudes in Green State of Denmark: Implications for Energy Democracy, Low Carbon Transitions, and Energy Literacy. Environmental Science and Policy 54: 304-315 (2015)

9. Brounen, D.; Kok, N.; Quigley, J.M.: Energy Literacy, Awareness, and Conservation Behavior of Residential Households. Energy Economics 38, 42-50 (2013)

10. Attari, S.Z.; DeKay, M.L.; Davidson, C.I.; Bruine de Bruin, W.: Public Perceptions of Energy Consumption and Savings. Proceedings of the National Academy of Sciences of the United States of America 107(37), 16054-16059 (2010)

11. Niemelä, R.; Ek, S.; Eriksson-Backa, K.; Huotari, M.-L.: A Screening Tool for Assesing Everyday Health Information Literacy. Libri 62(2), 125-134 (2012)

12. Hirvonen, N., Enwald, H., Nengomasha, C., Abankwah, R., Uutoni, W.U., Korpelainen, R., Pyky, R., Huotari, M.-L., Mayer, A.-K. Validating the Factorial Structure of the Everyday Health Information Literacy Screening Tool in Three Different Populations. ISIC: the Information Behvaiour Conference, University of Zadar, Croatia, September 20-23. Extended abstract for a poster (2016)

13. Enwald, H.; Hirvonen, N.; Huotari, M.-L.; Korpelainen, R.; Pyky, R.; Savolainen, M.; 
Salonurmi, T.; Keränen, A-M.; Jokelainen, T.; Niemelä, R.: Everyday Heath Information Literacy among Young Men Compared to Adults with High Risk for Metabolic Syndrome a Cross-Sectional Population-Based Study. Journal of Information Science 42(3), 344-355 (2016)

14. Cotton, D.R.E.; Miller, W.; Winter, J.; Bailey, I.; Sterling, S.: Developing Students' Energy Literacy in Higher Education. International Journal of Sustainability in Higher Education 16(4), 456-473 (2015)

15. Hirvonen, N.; Niemelä, R.; Ek, S.; Korpelainen, R.; Huotari, M-L.: Socio-Demographic Characteristics Associated with the Everyday Health Information Literacy of Young Men. Information Research 20(1), paper: isic25 (2015) 